Ann. Biol. anim. Bioch. Biophys., I964, 4 (I), 35-47.

\title{
ÉVOLUTION DE LA COMPOSITION CORPORELLE CHEZ LE RAT BLANC EN CROISSANCE
}

\author{
A. RÉRAT, C. FÉVRIER, Y. HENRY, J. LOUGNON \\ avec la collaboration technique de Yvonne Danied, Françoise Houl./er, Marie-Claire Tieron et G. Rolzet \\ Station de Recherches sur l'Élevage des Porcs, \\ Centre national de Recherches zootechniques, Jouv-en-Josas (Seine-et-Oise)
}

SOMMAIRE

La composition corporelle de 158 rats blancs Wistar $\mathrm{CF}$, nourris ad libitum d'un régime à base de caséine et dont le poids varie entre 35 et $260 \mathrm{~g}$, a été déterminée afin de connaître l'évolution de chacun des constituants corporels (eau, azote, cendres, lipides) en fonction du poids vif. La quantité de chacun de ces composants présents dans l'organisme évolue selon une fonction exponentielle du poids vif. Cette évolution n'est pas la même selon le constituant envisagé : lorsque le poids vif croît, le pourcentage d'eau et de minériaux décroît, le pourcentage d'azote augmente légèrement, le pourcentage de lipides s'élève dans des proportions notables. Crâce aux équations de régression obtenues, il est possible de calculer aver une précision de $5 \mathrm{p}$. Ioo le contenu azoté initial des animaux destinés à l'expérimentation et, par là même, de mesurer la rétention azotée selon la méthode de Rosfnibera (1959) par le seul dosage de l'azote final.

\section{INTRODUCTION}

Les méthodes proposées pour mesurer la valeur nutritive des protéines ont fait l'objet de nombreuses revues critiques (CHICK, I942; MitchelL, I944; AlLISON, I949, I955; ADRIAN et RÉRAT, I958 ; BENDER, I958). L'une de ces méthodes, basée sur 1'analyse chimique corporelle (Mc Collum et Simmonds, I929; RosenBERG, I959) présente, entre autres avantages, celui de la simplicité. La critère utilisé est, dans ce cas, la rétention azotée calculée à partir de la différence entre les quantités d'azote présentes dans le corps à la fin et au début de la période expérimentale, au cours de laquelle l'animal a reçu la protéine étudiée. S'il est facile de connaître la composition corporelle des animaux après qu'ils aient subi les traitements expérimentaux, il est évidemment impossible de mesurer directement le contenu azoté de l'organisme de ces animaux avant le début de l'expérience. La quantité d'azote initiale peut cependant être déduite de l'analyse corporelle d'animaux témoins 
soumis aux mêmes conditions préexpérimentales que les autres animaux. Deux possibilités s'offrent alors :

- soit sacrifier au début de chaque expérience un certain nombre d'animaux représentatifs de l'ensemble de la population utilisée,

- soit déterminer, une fois pour toutes, sur un grand nombre d'animaux soumis au régime préexpérimental les lois qui règlent l'évolution de la composition corporelle et les facteurs qui la font varier.

C'est cette deuxième solution que nous avons retenue : la présente étude a pour objet d'examiner les variations de la composition corporelle en fonction du poids vif chez le Rat en croissance soumis à un régime donné qu'il ingère ad libitum.

\section{MATÉRIEL, E'T MÉTHODE}

Trois groupes de rats mâles de souche Wistar pris au sevrage, représentant au total I 58 animaux, ont été utilisés successivement pour cette étude. Les animaux sont placés dans des cages individuelles et reçoivent ad libitum, pendant une période variable, un régime préexpérimental dont la composition est donnée dans le tableau I.

\section{Tableau I}

Composition du régime préexpérimental

$\begin{array}{r}(\mathrm{g}) \\ -120 \\ 3 \\ 40 \\ 20 \\ 80 \\ 727 \\ 10 \\ \hline 1000\end{array}$

(1) Composition du mélange vitaminique :

Thiamine (chlorhydrate) $\ldots \ldots \ldots \ldots \ldots \ldots \ldots \ldots \ldots \ldots \ldots \ldots \ldots \ldots$

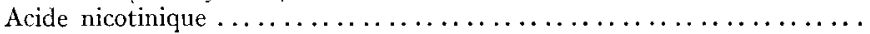

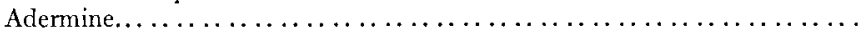

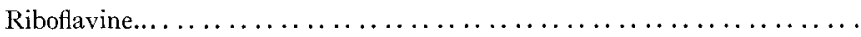

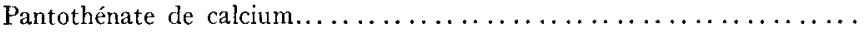

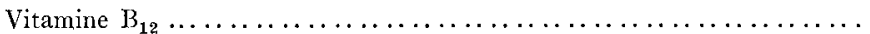

Acide folique $\ldots \ldots \ldots \ldots \ldots \ldots \ldots \ldots \ldots \ldots \ldots \ldots \ldots \ldots \ldots \ldots \ldots \ldots$

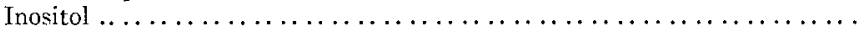

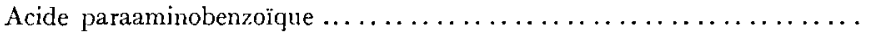

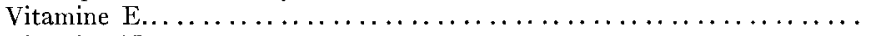

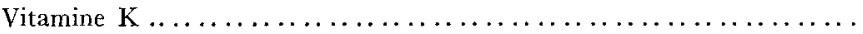

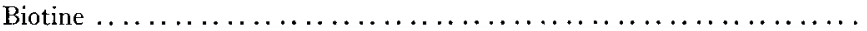

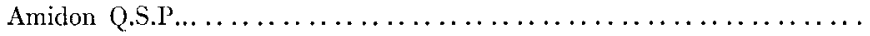

(mg)
-
8
30
8
15
30
0,03
1
500
500
30
5
0,2
$10 \mathrm{~g}$

Les vitamines $\mathrm{A}$ et $\mathrm{D}$ sont administrées dans l'huile d'arachide sous forme commerciale (4 $\mathrm{ml}$ de "Vitadone "Byla pour I $000 \mathrm{ml}$ d'huile).

La choline est ajoutée au régime à raison de $5 \mathrm{ml}$ d'une solution à $20 \mathrm{p}$. 100 par kilogramme de régime. 
Les poids, au jour du sacrifice, sont compris entre 35 et roo g pour les 54 animaux du premier groupe, entre 60 et $200 \mathrm{~g}$ pour les 64 animaux du deuxième groupe, entre $\mathrm{I} 20$ et $260 \mathrm{~g}$ pour les 40 animaux du troisième groupe.

Vingt-quatre heures après la distribution du dernier repas, les animaux sont sacrifiés par asphyxie au gaz d'éclairage. Les carcasses, placées dans des sacs en film plastique, sont entreposées à la chambre froide à - I $5^{\circ} \mathrm{C}$. La durée de conservation peut ainsi atteindre une année sans que le poids des animaux diminue de plus de $0,3 \mathrm{~g}$.

Dès la sortie de la chambre froide, la carcasse entière est sectionnée en plusieurs fragments et broyée dans un hachoir à viande (Bauknecht); puis, elle est finement hachée à l'aide d'un masticateur jusqu'à l'obtention d'un broyat homogène. Des prises aliquotes (3 à $6 \mathrm{~g}$ ) sont prélevées sur ce broyat en vue d'effectuer le dosage de l'humidité, de l'azote et des cendres. La matière sèche est obtenue par dessication à $104^{\circ} \mathrm{C}$ jusqu'à poids constant atteint en $4^{8} \mathrm{~h}$ environ. On procède alors à l'incinération de l'échantillon sec pendant $36 \mathrm{~h}$ à $55^{\circ} \mathrm{C}$. L'azote est dosé par la méthode Kjeldahl avec fixation de $\mathrm{NH}_{3}$ par l'acide borique. Chaque dosage est effectué en triple exemplaire. Les lipides sont calculés par différence entre le poids total et la somme (eau + cendres + protéines $(N \times 6,25)$ ) ; les glucides ne représentent en effet qu'une part négligeable dans la composition corporelle (MickELSEN et ANDERSON, I959) ; de toute façon, un test comparatif (HENRY et RÉRAT, 1963) nous a permis de vérifier que cette méthode d'estimation des lipides par différence donne des résultats très voisins ( 3 p. Ioo par excès) de ceux obtenus par la technique de dosage au chloroforme préconisée par BLIGH et DYER (1959).

\section{RÉSULTATS}

Les quantités des différents constituants chimiques présents dans l'organisme (eau, azote, cendres, lipides) ont été mises en relation soit avec le poids vif, soit avec le poids dégraissé, soit entre elles.

\section{TABLEAU 2}

Relations des constituants chimiques de l'organisme entre eux et vis-à-vis du poids de l'animal

\begin{tabular}{|c|c|c|c|}
\hline Y & $\mathrm{X}$ & $\begin{array}{l}\text { Équation de régression } \\
\qquad \log \mathrm{Y}=\end{array}$ & $\begin{array}{c}\text { Coefficient } \\
\text { de corrélation } r\end{array}$ \\
\hline $\begin{array}{l}\text { Eau }(g) \ldots \ldots \ldots \ldots \\
\text { Azote }(g) \ldots \ldots \ldots \ldots \\
\text { Cendres }(g) \ldots \ldots \ldots \\
\text { Lipides }(g) \ldots \ldots \ldots\end{array}$ & $\begin{array}{l}\text { Poids vif }(g) \\
\text { Poids vif }(\mathrm{g}) \\
\text { Poids vif }(\mathrm{g}) \\
\text { Poids vif }(\mathrm{g})\end{array}$ & $\begin{array}{l}-0,0051+0,921( \pm 0,007)(1) \log X \\
-1,5815+1,024( \pm 0,017) \log X \\
-1,3052+0,923( \pm 0,033) \log X \\
-2,1803+1,567( \pm 0,069) \log X\end{array}$ & $\begin{array}{l}0,9988 \\
0,9944 \\
0,9768 \\
0,9551\end{array}$ \\
\hline $\begin{array}{l}\text { Eau }(g) \ldots \ldots \ldots \ldots \\
\text { Azote }(g) \ldots \ldots \ldots \ldots \\
\text { Cendres }(g) \ldots \ldots \ldots \ldots\end{array}$ & $\begin{array}{l}\text { Poids dégraissé }(g) \\
\text { Poids dégraissé }(g) \\
\text { Poids dégraissé }(g)\end{array}$ & $\begin{array}{l}-0,0773+0,978(-0,005) \log X \\
-1,6644+1,089(-0,015) \log X \\
-1,3841+0,983(\div 0,031) \log X\end{array}$ & $\begin{array}{l}0,9995 \\
0,9962 \\
0,9808\end{array}$ \\
\hline $\begin{array}{l}\text { Eau }(g) \ldots \ldots \ldots \ldots \\
\text { Azote }(g) \ldots \ldots \ldots \ldots\end{array}$ & $\begin{array}{l}\text { Azote }(\mathrm{g}) \\
\text { Cendres }(\mathrm{g})\end{array}$ & $\begin{array}{r}-1,5715+1,109( \pm 0,020) \log X \\
0,1183+0,905( \pm 0,024) \log X\end{array}$ & $\begin{array}{l}0,9935 \\
0,9865\end{array}$ \\
\hline$\frac{\text { Azote }}{\text { Eau }}$ & Poids vif $(\mathrm{g})$ & $-1,5764+0,103 \log \mathrm{X}$ & \\
\hline
\end{tabular}

(1) L'intervalle de confiance du coefficient de régression a été estimé au seuil 0,05 . 
$\left.\mathrm{I}^{\circ}\right)$ Relations entre la composition corporelle et le poids vif

(tableau 2, graphiques I et 2)

Nous avons représenté graphiquement, en coordonnées logarithmiques, les quantités d'azote, de lipides, de minéraux et d'eau contenues dans l'organisme en fonction du poids des animaux (graph. I et 2). Pour chactun des constituants, les points se répartissent sur une même droite, ce qui signifie que leur développement suit une allure exponentielle en fonction du poids. L'ajustement des données ainsi transfor-

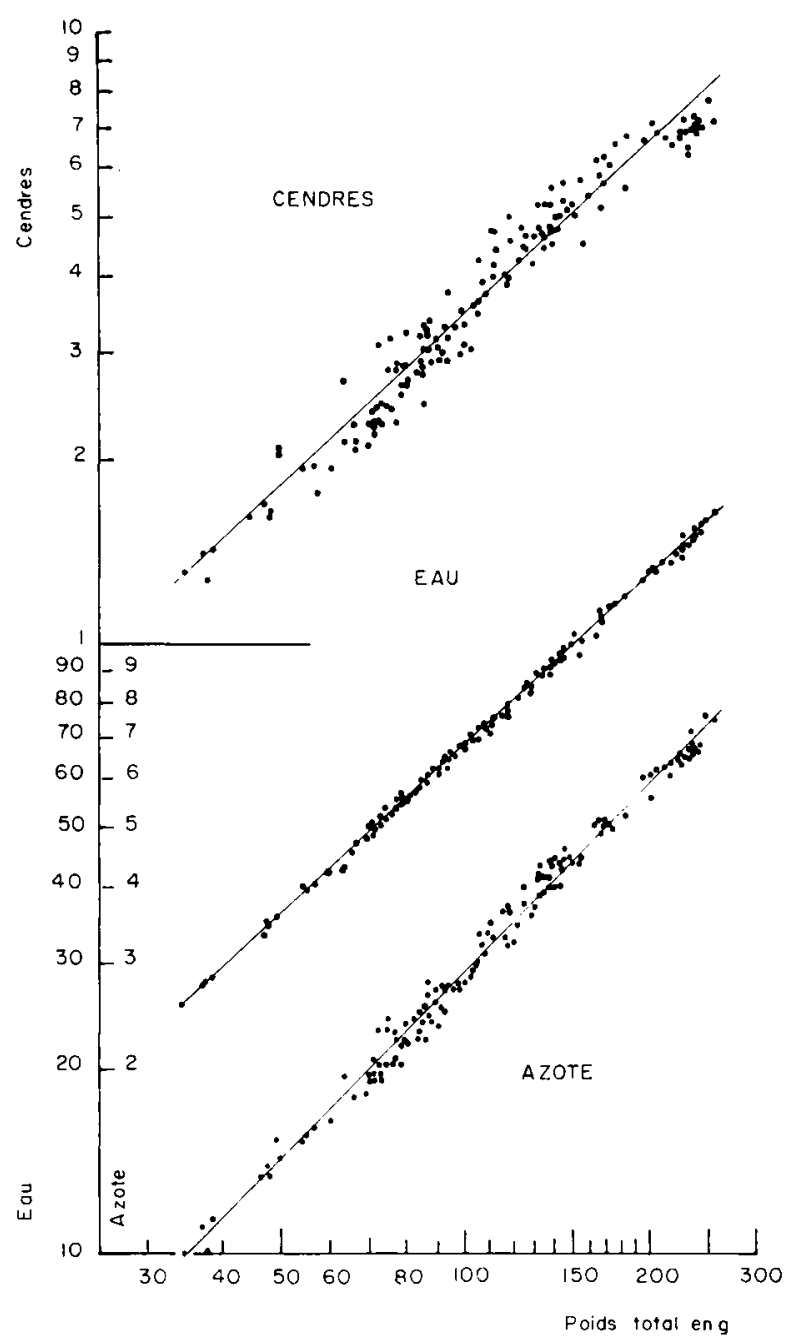

GRAPH. I. - Relations entre les constituants corporels et le poids vif

mées, suivant la méthode des moindres carrés, a permis d'obtenir, pour la gamme de poids étudiés (30-260 g), une série d'équations linéaires de la forme :

$$
\log \mathrm{X}=\log a+b \log \mathrm{P},
$$


$\mathrm{X}$ étant la quantité de constituants pour un poids $\mathrm{P}$ donné, $a$ et $b$ des constantes caractéristiques de la régression et différentes selon la nature du dépôt. Les relations précédentes peuvent également s'écrire sous la forme exponentielle :

$$
\mathrm{X}=a \cdot \mathrm{P}^{b} \text {. }
$$

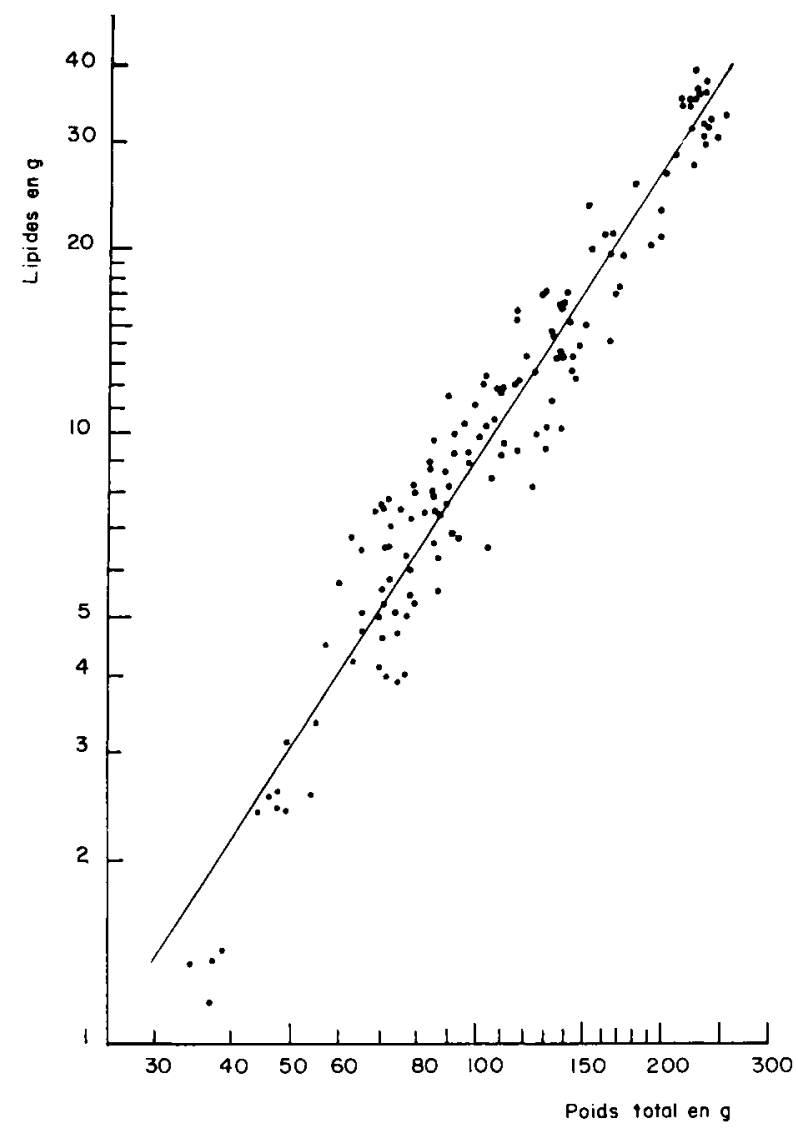

GRAPH. 2. - Relation entre les lipides corporels et le poids vif

Des valeurs de l'exposant du poids dans ces diverses équations, suivant qu'elles sont inférieures ou supérieures à l'unité, il est possible de déduire les faits suivants : lorsque le poids vif croît, le pourcentage d'eau et de minéraux dans l'organisme décroît, le pourcentage d'azote reste pratiquement invariable ou augmente légèrement, le pourcentage de lipides s'élève dans des proportions notables. C'est ainsi qu'entre 30 et $260 \mathrm{~g}$ de poids vif, les valeurs de ces pourcentages passent respectivement, pour l'eau de 74,3 à 63,0 p. Ioo, pour les minéraux de 3,7 à 3,2 p. Ioo, pour les matières azotées de I7,5 à I $8,5 \mathrm{p}$. IOO, et pour les lipides de 4,5 à I5,3 p. IOO (graph. 3). Il faut noter que si les proportions d'azote et d'eau présentes dans l'organisme varient relativement peu d'un animal à l'autre, il n'en est pas de même pour les quantités de minéraux et surtout de lipides qui présentent des variations 
de grande amplitude, comme le montrent le graphique 2 et la valeur de l'intervalle de confiance du coefficient de régression (tabl. 2 ).

\section{0) Évolution de la composition du gain de poids}

A partir des équations précédentes, il est possible de calculer la composition du gain de poids (graph. 3). Il ressort de ce graphique que les constituants qui varient essentiellement dans les tissus formés au cours de la croissance sont l'eau et les lipides qui subissent une évolution divergente. Par ailleurs, dans le cas du régime utilisé, le dépôt de lipides par unité de gain de poids dépasse celui des protéines lorsque le poids de l'animal atteint $200 \mathrm{~g}$.

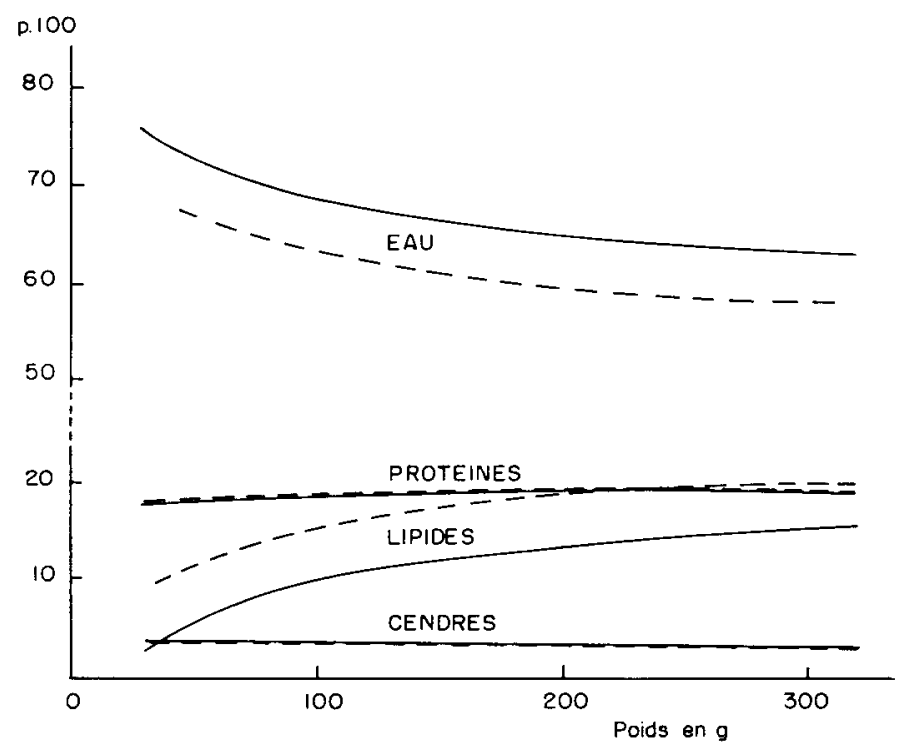

GRAPH. 3. - Évolution de la composition corporelle et du gain de poids

- poids vif

$\left.3^{\circ}\right)$ Relations entre la composition corporelle et le poids de l'animal dégraissé

Compte tenu de la forte variabilité de la teneur en lipides des rats pour un poids donné, il a paru intéressant de rechercher la forme des relations entre la quantité des constituants chimiques de l'organisme et le poids du corps débarrassé des lipides. Ainsi que l'on pouvait s'y attendre, les équations obtenues (tabl. 2), qui sont illustrées dans le graphique 4 , sont de la même forme que précédemment. La variabilité des données ayant permis de les obtenir est beaucoup moins élevée et les coefficients de corrélation sont, en conséquence, plus élevés que dans les relations avec le poids vif. Il faut noter que la pente des diverses droites se rapproche de l'unité, ce qui signifie que la composition du corps délipidé ne varie que dans de très faibles proportions. 
Ainsi, entre 30 et $260 \mathrm{~g}$ de poids vif, le pourcentage d'eau dans les tissus dégraissés passe de 77,8 à 74,4 , celui des matières azotées de $\mathrm{I} 8,2$ à $2 \mathrm{I}, 8$ et celui des minéraux de 3,9 à 3,8 .

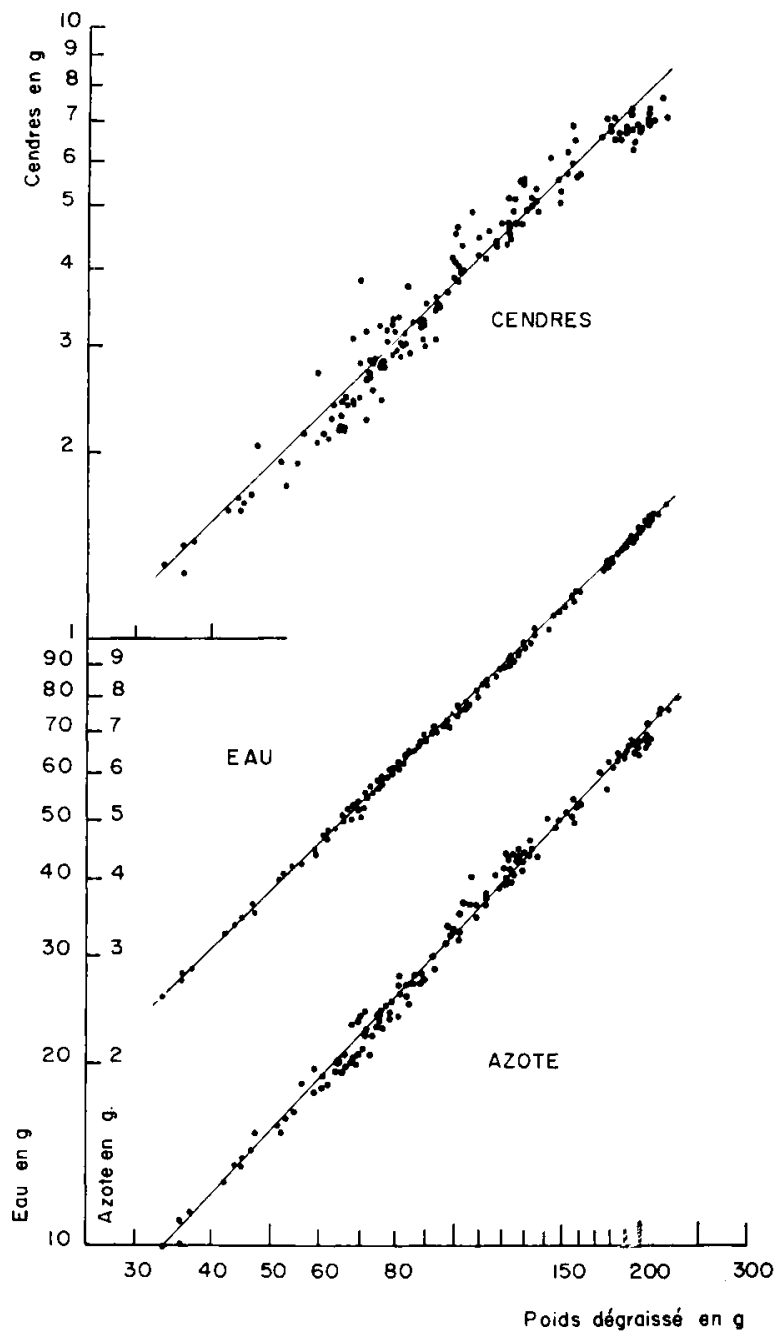

Grapri. 4. - Relations entre les constituants corporels et le poids dégraissé

$\left.4^{\circ}\right)$ Relations entre les différents constituants

Les relations entre les composants de la masse corporelle dégraissée sont rapportées dans le tableau 2 et le graphique 5 .

La proportion des minéraux, par rapport à l'azote, diminue lorsque augmente le poids du corps ; par contre, la pente de la droite de régression de l'azote sur l'eau est supérieure à l'unité, ce qui montre que le rapport $\mathrm{N} / \mathrm{H}_{2} \mathrm{O}$ s'accroît dans le même temps. 
L'étude du rapport $\mathrm{N} / \mathrm{H}_{2} \mathrm{O}$ est particulièrement intéressante, car c'est en tenant compte de l'évolution de ce rapport qu'ont été fixées les modalités d'une méthode d'évaluation de la valeur nutritive des protéines (BENDER et MILLER, I953). A partir

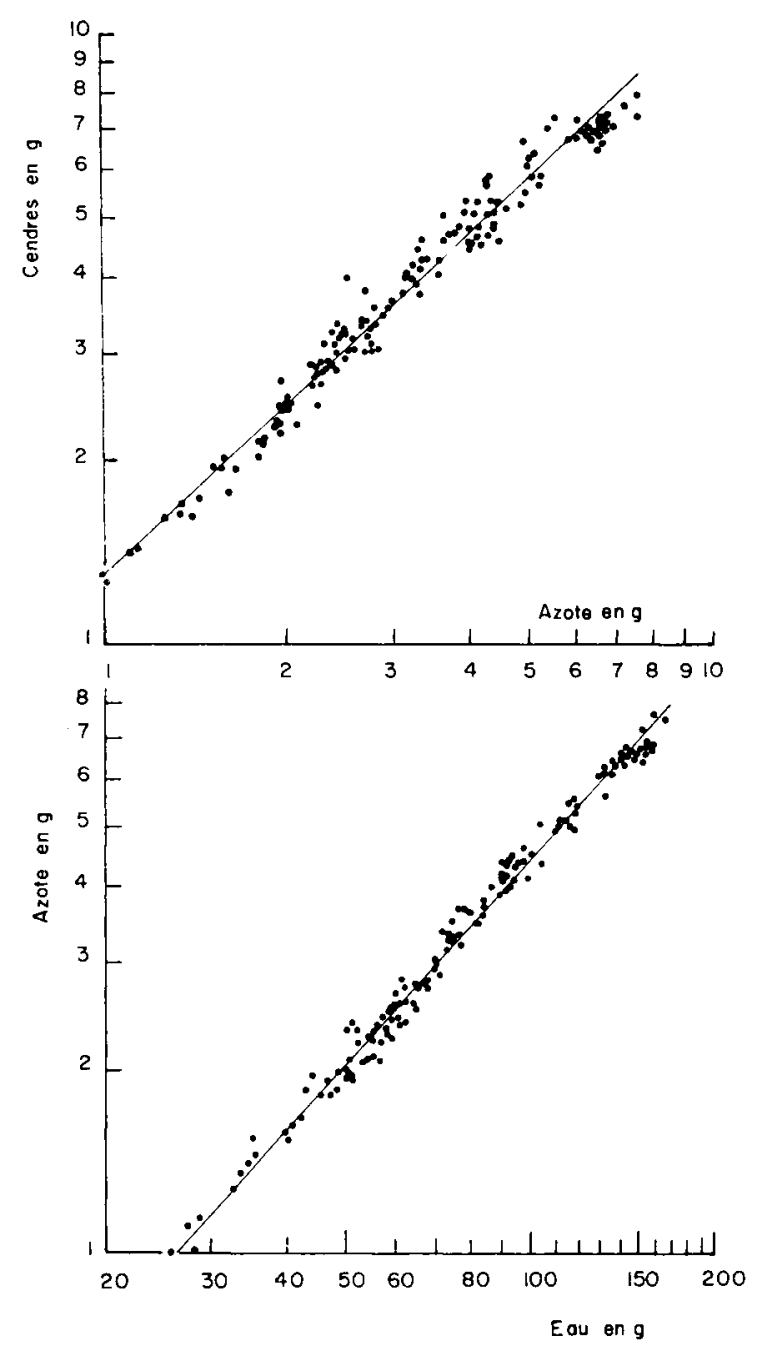

GRAPH. 5. - Relations entre différents constituants corporels

des équations précédentes, il est possible d'établir que ce rapport suit une évolution exponentielle en fonction du poids vif, évolution traduite par l'équation :

$$
\frac{\mathrm{N}(\mathrm{g})}{\mathrm{H}_{2} \mathrm{O}(\mathrm{g})}=0,38 \cdot \mathrm{P}^{0,103}
$$




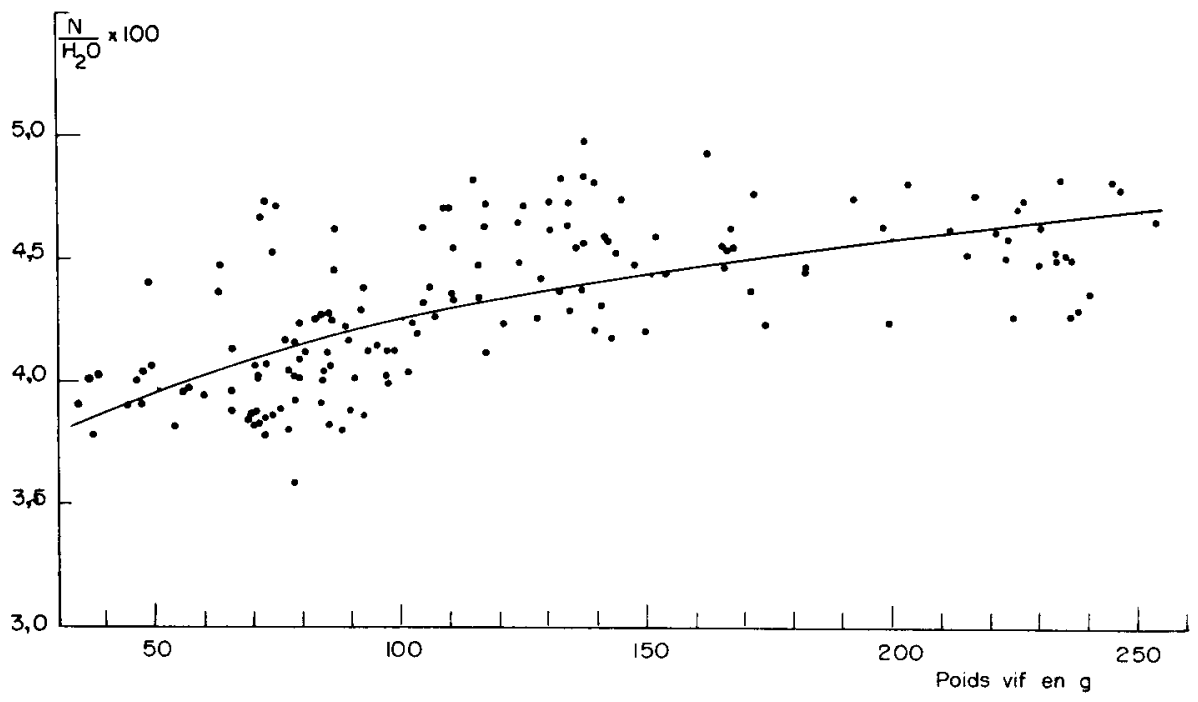

GRAPH. 6. - Évolution du rapport $\mathrm{N} / \mathrm{H}_{2} \mathrm{O}$ en fonction du poids

\section{DISCUSSION}

Les phénomènes de croissance différentielle ont été mis à maintes reprises en évidence chez différentes espèces et pour différents organes, tissus ou constituants chimiques (Brody, I945; HAMMOND, I932; MCMEEKAN, I940 ; PALSSON, I955; Palsson et Verges, I952 ; Cuthberson et Pomeroy, I962).

Nos propres résultats pour 1'essentiel confirment cette notion bien établie. Ils présentent, cependant, vis-à-vis des données d'autres auteurs des différences de détail portant en particulier sur la pente des droites de régression obtenues; ces différences peuvent être expliquées autant par des causes spécifiques que par des raisons alimentaires. Ainsi, à l'appui des résultats obtenus au cours de la présente étude, d'autres travaux ont mis en évidence l'accroissement de la concentration en matières protéiques des tissus formés en fonction du poids de l'animal, chez le Rat (Forbes et RAo, I959) et chez la Souris (BAILEy et al., Ig6o). Cependant la la pente de la droite de régression mettant en relation protéines corporelles et poids de l'animal est de I,I6 chez la souris contre I,05 dans nos expériences. A l'inverse, Hogan (I925), Clausen (I953), Jespersen (I952) signalent que chez le Porc le pourcentage d'azote dans les tissus diminue au fur et à mesure que le poids s'élève ; c'est également ce que nous avons pu montrer récemment (RÉRAT et HENRY, Ig63 b). Ėnfin, selon WENIGER (I955) la concentration d'azote dans les tissus formés serait d'autant plus élevée pour un poids donné que le régime est plus riche en matières protéiques.

En ce qui concerne l'évolution de la teneur en minéraux des tissus, lorsque augmente le poids, elle semble diminuer chez le Rat comme cela a été également signalé chez le Porc (HoGan, I925), alors qu'elle augmente très fortement chez la Souris (BAILEY et al., I960); il faut noter, ici encore, que la teneur en minéraux du 
corps semble dépendre de la nature du régime : elle est sensiblement plus élevée chez les porcs recevant des régimes à faible taux azoté que chez des animaux recevant des régimes à taux azoté élevé (WENIGER, I955). Quoi qu'il en soit, compte tenu de l'évolution inverse des minéraux et de 1'azote dans les tissus des animaux soumis à nos expériences, il semble difficile de conclure à la constance de composition de la matière sèche corporelle délipidée admise par MurRay (I922), BAILEY et al. (I960) et Clawson et al. (I955); on peut admettre cependant que les variations observées sont de faible amplitude (MOUlton, I923; PACE et RATHBUn, I945 ; SpRay et Widdowson, I950; MCCANCE et Widdowson, I956).

En ce qui concerne les lipides et l'eau, il est bien connu qu'ils représentent les composants variables de l'organisme et subissent une évolution. divergente : lorsque augmente le poids, les tissus formés sont de plus en plus chargés de lipides et contiennent de moins en moins d'eau (Moulton, I923; Oslage, I962 ; Osinska, I962). Il en résulte que le rapport $\mathrm{N} / \mathrm{H}_{2} \mathrm{O}$ a tendance à augmenter lorsque l'animal grandit : précisons à ce sujet que les valeurs que nous obtenons sont légèrement supérieures à celles déduites de l'application de la formule de MILLER et BENDER (I955), équation obtenue d'après des données corporelles de I 97 rats de souche différente et de o à $503 \mathrm{j}$ d'âge, mais elles se rapprochent de celles obtenues par LIGHT et al. (I934).

Le contenu lipidique du corps présente en outre une grande variabilité (CLAWSON et al., I955; BAILEY et al., I960; LASSITER et al., I960), liée pour une part à la composition du régime (WENIGER, I955) et au niveau d'alimentation (MCMEEKAN, I940). Notons, par exemple, que la pente de la droite de régression de la quantité de lipides corporels sur le poids vif est beaucoup plus élevée dans le cas ici décrit (régime à la caséine, à ro $\mathrm{p}$. Ioo de protéines), que dans le cas d'animaux recevant des régimes en alimentation séparée (HENRY et RÉRAT, I963). Cette variabilité pourrait être également liée au fait que la teneur lipidique des carcasses est généralement calculée par différence et non pas obtenue par dosage direct, sauf dans certains cas. On pourrait évidemment penser que cette façon de faire conduit à cumuler les erreurs des autres dosages au niveau de celui des lipides; cependant, compte tenu de la vérification faite sur une dizaine de carcasses, l'erreur systématique de $3 \mathrm{p}$. Ioo par excès due à l'application de la méthode par différence vis-à-vis du dosage direct ne saurait être la cause de la variabilité de la teneur lipidique ; en tout état de cause, elle peut être attribuée aux éléments non dosés de la carcasse, en particulier le glycogène du foie et des muscles.

De l'ensemble de ces faits, il résulte que l'on peut prévoir aisément la composition chimique de l'organisme d'animaux recevant un régime donné. Cela ne préjuge en rien des modifications pouvant survenir lorsque varient la nature du régime et le niveau de consommation et pour lesquelles, malgré quelques études récentes (KROPF et al., I959; WENIGER, I955; OSLAGE, I962, I963 $a$ et $b$ ), il subsiste encore de nombreuses inconnues. Sur le plan pratique, si l'on veut appliquer cette prévision au calcul de la composition corporelle initiale d'animaux soumis ensuite à des expériences de plus ou moins longue durée, il est bon de connaître l'importance de l'erreur que cette méthode amènera à commettre sur les rétentions azotée et calorique. La formule générale permettant d'obtenir la valeur de ces rétentions (ROSENBERG, I959) est la suivante :

Quantité d'élément retenue $=$ quantité présente dans l'organisme à la fin de l'expérience $\mathrm{C}_{2}$ - quantité présente dans l'organisme au début de l'expérience $\mathrm{C}_{\boldsymbol{1}}$. 
Il est évident que l'erreur portant sur $\mathrm{C}_{1}$ aura d'autant moins d'importance relative que $\mathrm{C}_{2}$ sera plus élevé. En ce qui concerne l'azote, l'application de l'équation de régression obtenue permet de déduire que le contenu de la carcasse sera de I,I57 $\pm 0,055 \mathrm{~g}$ (pour un seuil de probabilité 0,05 ) chez un rat de $40 \mathrm{~g}$ et de $\mathrm{I}, 708$ $\pm 0,077 \mathrm{chez}$ un rat de $60 \mathrm{~g}$, soit une erreur relative variable oscillant aux environs de 5 p. roo pour des animaux de ces poids. Rappelons que l'erreur commise sur $\mathrm{C}_{2}$ par dosage direct de l'azote est d'environ $2 \mathrm{p}$. Ioo ; la quantité d'azote finale étant fonction du poids en fin d'expérience et celui-ci étant lié à la durée de l'expérience et à la valeur nutritive du régime expérimental, la part de l'erreur initiale dans l'erreur totale est évidemment variable : elle est d'autant plus grande que la protéine utilisée est plus déséquilibrée et que le test a duré moins longtemps. A titre d'exemple, les erreurs commises chez des rats de $55 \mathrm{~g}$ soumis à une expérience de 56 jours et présentant une croisiance quotidienne de 5,I7 g (RÉRAT et HENRY, I963a) sont pour l'azote respectivement de $0,2 \mathrm{I} 4 \mathrm{~g}$ pour $\mathrm{C}_{2}$ et $0,072 \mathrm{~g}$ pour $\mathrm{C}_{1}$, soit une erreur totale de $0,286 \mathrm{~g}$ pour une rétention de 9 , II $\mathrm{g}$; ces erreurs sont respectivement de $0,09 \mathrm{I} \mathrm{g}$ et $0,069 \mathrm{~g}$ pour des rats ayant présenté une croissance de $\mathrm{I}, 89 \mathrm{~g}$ par jour, soit une erreur totale de $0, \mathrm{I} 60 \mathrm{~g}$ pour une quantité retenue de $3,03 \mathrm{~g}$. Les erreurs relatives correspondantes sont de 3 , I $\mathrm{p}$. Ioo pour un gain de 5 , I7 $\mathrm{g} / \mathrm{j}$ et de $5,3 \mathrm{p}$. Ioo pour un gain de $\mathrm{I}, 89 \mathrm{~g} / \mathrm{j}$.

En ce qui concerne les lipides, l'erreur sur la composition corporelle initiale due à l'application des équations de régression est plus importante et se situe aux environs de 15 à 20 p. IOO.

En conclusion, 1'évolution de la composition corporelle de rats soumis à un régime donné suit des règles précises qui permettent de prévoir le contenu en azote, cendres, eau et lipides de ces animaux à un poids donné. En effet, la quantité de chacun des composants présents dans l'organisme évolue selon une fonction exponentielle du poids vif. Lors de l'application de la méthode de Rosenberg (I959) pour le calcul de la rétention azotée par analyse corporelle, la quantité d'azote présente dans le corps au début de l'expérience peut ainsi être estimée à partir des équations de régression obtenues.

Reçu pour publication en janvier 1964 .

\author{
SUMMARY
}

CHANGES JN BODY COMPOSITION IN THE WHITE RAT DURING GROWTH

From weaning 158 Wistar $C F$ white rats were fed to appetite on a diet based on casein (table $\mathrm{r}$ ). They were killed at liveweights ranging from 35 to $260 \mathrm{~g}$ and body composition was estimated to study the quantitative changes in each of the constituents of the body (water, nitrogen, ash and lipids) in relation to liveweight. By logarithmic transformation of the results it was possible to formulate regression equations relating each of the body constituents to liveweight and to fat-free bodyweight, and also relating the different constituents to each other.

The change in the amount of a constituent of the body in relation to live weight varies, depending on the constituent under consideration (graphs I and 2). Thus, as liveweight increases the proportions of water and mineral decrease, while that of nitrogen increases to a small extent and that of fat rises rapidly. These changes are even more marked in the composition of the weight gained 
(graph 3). Since there is wide variation in the lipid content, the amounts of water, nitrogen and ash have been related to fat-free weight (graph 4): the differences in composition on this basis are of much smaller amplitude. The relations between ash and nitrogen and between nitrogen and water have been calculated : the ratio $\frac{\mathrm{N}}{\mathrm{H}_{2} \mathrm{O}}$ increases exponentially with liveweight.

With the regression equations obtained it is possible to calculate, with an accuracy within 5 p. 100, the initial nitrogen content of animals at the beginning of the experiment, and from what even to measure retention of nitrogen according to the method of ROSENBERG (1959), from the single estimation of nitrogen content at the end.

\section{RÉFÉRENCES BIBI,IOGRAPHIQUES}

ADRIAN J., RÉrat A., 1958. Méthodes d'évaluation de la valeur nutritive des protéines. C. N. R. S., Paris.

Allison J. B., i949. Biological evaluation of proteins. Adranc. Protein. Chem., 5, 155-200.

Atrison J. B., 1955. Biological evaluation of proteins. Physiol. Rev., 35, 664-700.

BAILEY C. B., KITTS W. D., WooD A. J., ig60. Changes in the gross chemical composition of the mouse during growth in relation to the assessment of physiological age. Canad. J. Anim. Sci., 40, I43-I 55.

Bender A. E., 1958. Biological methods of evaluating protein quality. Proc. Nutr. Soc., 17, 85-9o.

Bender A. E., Miller D. J., I953. Constancy of the $\mathrm{N} / \mathrm{H}_{2} \mathrm{O}$ ratio of the rat and its use in the determination of the net protein value. Biochem. I., 53, VII-VIII.

Bligh E. G., Dyer W. J., I959. A rapid method of total lipid extraction and purification. Canad. J Biochem. Physiol., 37, 911-9I7.

Brody S., I945. Bioenergetics and growth. Reinhold publishing corporation, New York.

Chick H., 1942. Biological value of the proteins contained in wheat flours. Lancet, 242, 405-408.

Clausen H., I953. The improvement of pigs. The George Scott Robertson Memorial Lect.

Clawson A. J., Sheffy B. E., Reid J. T., t955. Some effects of feeding chlortetracycline upon the carcass characteristics and the body composition of swine and a scheme for the resolution of the body composition. I. Anim. Sci., 14, I122, 1 132 .

Cuthberson A., Pomeroy R. W., 1962. Quantitative anatomical studies of the composition of the pig at 50,68 and $92 \mathrm{~kg}$ carcass weight. II -- Gross composition and skeletal composition. J. Agric. Sci., 59, $215-223$.

Forbes R. M., Rao T., I959. The effect of age on the net requirements for Nitrogen, Lysine and Tryptophan by the well-fed rat. Arch. Biochem. Biophys., 82, 348-354.

Hammond J., 1932. Growth and the development of mutton qualities in the sheep. Oliver et Boyd, Edinburgh.

Henry Y., Rérat A., 1963. Étude de l'ingestion spontanée d'éléments énergétiques et de protéines chez le Rat en croissance par la méthode du libre choix. Ann. Biol. anim. Bioch. Biophys., 3, 103-II7.

Herschdoerfer S. M., Joln M. G., 1962. Studies on the composition of pig carcasses. Chem. Ind. 17, $769-770$.

Hogan A. G., et al. 1925. The relation of feed consumed to protein and energy retention. Mo. Agr. Exp. Sta. Bul., 73.

JESPERSEN J., I952. Übersicht über die Arbcit der Staatlichen Tierzuchtversuchsanstalt im Jahre 1951-52, Kopenhague, I952. II-Abteilung für Schweineversuche. Züchtungskunde, 24, 229-230.

KROPF D. H., et al. 1959. Effect of protein level and quality in swine rations upon growth and carcass development. J. Anim. Sci., 18, 755-762.

Lassiter J. W., Culdison A. E., CarmoN J. L.. 1960. Variations in body composition of mice having the same average daily gain. J. Anim. Sci., 19, $769-772$.

LigirT E. L., et al., 1934. Inorganic salts in nutrition : changes in composition of the whole animal induced by a diet poor in salts. J. Brol. Chem., 107, 689-697.

McCance R. A., Widdowson E. X., 1956. The chemical structure of the body. Quart. J. Exp. Phy' siol., 41, I-I7.

McCollum E. V., Simmonds N., I920. The newer knoreledge of nutrition. 4th ed. McMillan, New-York.

McMeekan C. P., 1940. Growth and development in the pig, with special reference to carcass quality characteristics. J. Agr. Sci., 30, 276-569.

Mickelsen O., ANDERSON A. A., I959. A method for preparing intact animals for carcass analyses J. Lab. Clin. Med., 53, 282-29o.

Millek D. S., BENDER A. E., 1955. The determination of the net utilization of proteins by a shortened method. Bril. J. Nutr., 9, $3^{82-388 .}$ 
Mitchell II. H., 1944. Determination of the nutritive value of the proteins of food products. Ind. Eng. Chem. Anal, Ed., 16, 606-700.

Moulton C. R., I923. Age and chemical development in mammals. J. Biol. Chem., 57, 79-97.

Murray J. A., Ig22. The chemical composition of animal bodies. J. Agr. Sci., 12, 103-Iog.

Osinska Z., I962. Estimation of protein, chemical fat and energy content in pigs. Anim. Prod., 4, 39 I-398.

OsLAGE H. J., 1962. Untersuchungen über die Körperzusammensetzung und den Stoffansatz wachsender Mastschweine und ihre Beeinflussung durch die Ernährung. 2-- Körperzusammensetzung and Stoffansatz wachsender Mastschweine unter den Bedingungen normaler Eimährung. Zischr. Tierphysiol. Tzerern. $u$. Futtermittelk., 17, 357-382.

Oslage H. J., I963a. Untersuchungen über die Körperzusammensetzung und den Stoffansatz wachsender Mastschweine und ihre Beeinflussung durch die Ernährung. 3 - Einfluss einer eingeschränkten Energiezufuhrin zweiten Teil der Mastperiode auf Körperzusammensetzung und Stoffansatz wachsender Mastschweine. Ztschr. Tierphysiol. Tierern. $u$. Fittermitlelk., 18, 14-34.

OslaGe H. J., $1963 b$. Untersuchungen über die Körperzusammensetzung und den Stoffansatz wachsender Mastschweine und ihre Beeinflussung durch die Ernährung. 3 - Einfluss einer eingeschränkten Energiezufuhr im ersten Teil der Mastperiode auf Körperzusammensetzung und Stoffansatz wachsender Mastschweine. Ztschr. Tierphysiol. Tierern. u. Fattermiltelk., 18, 35-37.

Pace N., Ratibun F., 1945. Studies on body composition. III. The body water and chemically combined nitrogen content in relation to fat content. J. Biol. Chem., 158, 685.

Palsson H., 1955. Conformation and body composition in Hammond J. Progress in the physiology of farm animals, Butterworths, London.

PALSSON H., VERces J. B., I952. Effects of the plane of nutrition on growth and the development of carcass quality in lambs. J. Agr. Sci., 4., I-I 49 .

Rérat A., Henry Y., i963a. Besoin en lysine du Rat en croissance : principe d'une méthodologie et résultats expérimentaux. C. R. Ac. Sci., 25\%, 3045-3048.

Rérat A., Henry Y., ig63b. F́tude du besoin azoté chez le Porc en croissance. I - Utilisation de la farine de poisson à trois taux différents. Ann. Zootech., 12, (sous presse).

Rosenberc H. R., 1959. Amino acid supplementation of foods and feeds in AlbanesE A. A. Protein and amino acid nutrition, 381-417, Academic Press, New York.

SPRAY C. M., WIDDOWSON E. M., I950. The effect of growth and development on the composition of mammals. Brit. J. Nuir., 4, 332-353.

Weniger J. A., I955. Untersuchungen über den Nahrstoff-und Energieansat $z$ verschiedener Schweinerassen im Hinblick auf Lebendgewicht, 'Tageszunahme und Emährung. Arch. Tierernahrung, 4, $293-33$ I. 